\title{
Improving the Morphology of the Perovskite Absorber Layer in Hybrid Organic/Inorganic Halide Perovskite MAPbI 3 Solar Cells
}

\author{
I. J. Ogundana and S. Y. Foo \\ Department of Electrical and Computer Engineering, Florida Agricultural and Mechanical University, Tallahassee, FL 32310, USA \\ Correspondence should be addressed to S. Y. Foo; sfoo@fsu.edu
}

Received 20 January 2017; Accepted 29 March 2017; Published 3 May 2017

Academic Editor: Sundaram Senthilarasu

Copyright (C) 2017 I. J. Ogundana and S. Y. Foo. This is an open access article distributed under the Creative Commons Attribution License, which permits unrestricted use, distribution, and reproduction in any medium, provided the original work is properly cited.

\begin{abstract}
Recently, perovskite solar cells have attracted tremendous attention due to their excellent power conversion efficiency, low cost, simple fabrications, and high photovoltaic performance. Furthermore, the perovskite solar cells are lightweight and possess thin film and semitransparency. However, the nonuniformity in perovskite layer constitutes a major setback to the operation mechanism, performance, reproducibility, and degradation of perovskite solar cells. Therefore, one of the main challenges in planar perovskite devices is the fabrication of high quality films with controlled morphology and least amount of pin-holes for high performance thin film perovskite devices. The poor reproducibility in perovskite solar cells hinders the accurate fabrication of practical devices for use in real world applications, and this is primarily as a result of the inability to control the morphology of perovskites, leading to large variability in the characteristics of perovskite solar cells. Hence, the focus of research in perovskites has been mostly geared towards improving the morphology and crystallization of perovskite absorber by selecting the optimal annealing condition considering the effect of humidity. Here we report a controlled ambient condition that is necessary to grow uniform perovskite crystals. A best PCE of $7.5 \%$ was achieved along with a short-circuit current density of $15.2 \mathrm{~mA} / \mathrm{cm}^{2}$, an open-circuit voltage of $0.81 \mathrm{~V}$, and a fill factor of 0.612 from the perovskite solar cell prepared under $60 \%$ relative humidity.
\end{abstract}

\section{Introduction}

Organic-inorganic metal halide perovskites have recently shown great potential for application in solar cells with excitingly high performances with an up-to-date NREL-certified record efficiency of $22.1 \%$, as reported by KRICT/UNIST in November 2016. Perovskite materials are currently being investigated because of their low cost materials, low cost fabrication techniques, and ability to act as efficient lightabsorber layer for organic and inorganic photovoltaic applications. Perovskite absorber materials have the good optical, electrical, and semiconductor properties such as high diffusion length and high charge mobility. Another important factor that makes perovskite material highly sought after is the processing and manufacturing techniques; there are many of deposition techniques that can be used in the preparation of high quality thin films; these include one-step deposition, two-step sequential deposition, thermal evaporation, and vapor-assisted vacuum deposition techniques.
We have seen different studies focused on ways to control the morphology of perovskite films through proper selection of annealing conditions (temperature and humidity), perovskite precursor solution ratio, additives to side-chain of precursor solution, and solvent engineering. Huang et al. [1] earlier reported the optimal crystallization conditions for planar and mesoporous perovskite layer architectures as $\sim 100^{\circ} \mathrm{C}$ with annealing duration ranging from 30 to 120 minutes. Salim et al. [2] discussed the impact of morphology and device architecture on device performance in perovskitebased solar cells. Eperon et al. [3] presented a morphological control scheme for high performance, solution-processed planar heterojunction perovskite solar cells, while Gong et al. [4] proposed a controllable perovskite crystallization approach by water additive for high performance solar cells. Hsu et al. [5] proposed a two-step thermal annealing process to improve the morphology of spin-coated films for highly efficient perovskite hybrid photovoltaics, while Dualeh et al. [6] and Saliba et al. [7] investigated the effects of 
thermal processing protocol and annealing temperature on film morphology and crystallization of organic-inorganic hybrid perovskite solar cells. Tidhar et al. [8] discussed the crystallization of methyl ammonium lead halide perovskites and its implications in photovoltaic applications. Grancini et al. [9] also studied the impacts of the crystallization processes on the structural and optical properties of hybrid perovskite films. Liang et al. [10] presented additive enhanced crystallization of solution-processed perovskite for highly efficient planar heterojunction solar cells.

In order to determine the optimal crystallization conditions, different experiments and characterization techniques (such as Scanning Electron Microscopy, current densityvoltage measurements, and incident photon-to-current efficiency measurements) have been adopted so as to properly interpret the results from experiments and fabrication. After interpreting the results from characterization data, we observed that poor perovskite crystallites translate to low power conversion efficiencies and more pin-hole defects are observed in the perovskite film.

\section{Experimental Section}

2.1. Materials. Indium tin oxide coated with polyethylene terephthalate film (ITO/PET) with $12 \mathrm{ohm} / \mathrm{sq}$ surface resistivity, patterned indium tin oxide coated glass slide (with $8 \mathrm{ohm} / \mathrm{sq}$ surface resistivity), VWR microscope slides, poly(3,4-ethylenedioxythiophene)-polystyrene sulfonate (PEDOT:PSS) (1.3 wt.\% dispersion in water), PEDOT content 0.5 wt. $\%$ and PSS content 0.8 wt. $\%$, titanium oxide $\left(\mathrm{TiO}_{2}\right) 16$ wt.\%, 1,2-Dichlorobenzene (DCB), N,Ndimethylformamide (DMF), and titania paste (transparent) 19.0 wt.\%, drierite (with indicator, 6 mesh), 0.50 um Whatman filter paper, isopropyl alcohol, acetone, methylamine solution (33 wt.\% in absolute ethanol, $\mathrm{CH}_{3} \mathrm{NH}_{2}$ ), hydroiodic acid (57 wt.\% distilled, HI), lead(II) iodide (99.99\%), and conductive silver printing ink (Ag paste) with resistivity range 5-6 $\mu \Omega \mathrm{cm}$ were ordered from Sigma-Aldrich and used as received without further modification or purification.

2.2. Perovskite Precursor Synthesis. Preparing the precursor perovskite solution is the first step in order to produce a perovskite solution and perovskite film. Methylammonium iodide $\left(\mathrm{CH}_{3} \mathrm{NH}_{3} \mathrm{I}\right)$ was prepared by reacting methylamine $\left(\mathrm{CH}_{3} \mathrm{NH}_{2}\right)$ solution in absolute ethanol with hydroiodic acid (HI) (based on the steps and quantities stated in literature) [11]. To produce $\mathrm{MAPbI}_{3}$ precursor solution, methylammonium iodide (MAI) and $\mathrm{PbI}_{2}$ were dissolved in anhydrous N,N-dimethylformamide (DMF) in a 1:1 molar ratio, at $0.88 \mathrm{M}$ of each in a nitrogen-filled box to yield $0.88 \mathrm{M}$ perovskite precursor solution.

2.3. Perovskite Thin Film Preparation. The $\mathrm{MAPbI}_{3}$ films were prepared by spin-coating the derived precursor solution in a nitrogen-filled glovebox at $2500 \mathrm{rpm}$ for $40 \mathrm{~s}$ on top of PEDOT:PSS/ITO/PET substrate. The resulting film was then annealed at $85^{\circ} \mathrm{C}$ for about 2 hours in a controlled humidity chamber. The thickness of $\mathrm{MAPbI}_{3}$ perovskite film was controlled by varying the spin-speed and also using varying precursor concentrations.

2.4. Device Fabrication. The perovskite solar cell devices (PSCDs) were fabricated on ITO glass substrates; the ITO substrate was partly etched with $1 \mathrm{M} \mathrm{HCl}$ acid so as to correctly allow for electrode contact placement and prevent short-circuit. The ITO substrates were initially precleaned with cleansing soap and sonicated in deionized water, acetone, and isopropanol for 15 minutes. The substrates were dried in vacuum oven and thereafter allowed to undergo oxygen plasma treatment for 15 minutes. PEDOT:PSS solution was filtered using Whatman filter paper in order to remove aqueous colloidal particles in the original solution. The filtered PEDOT:PSS solution was spun on the ITO substrate at $2500 \mathrm{rpm}$ for 45 seconds and annealed at $120^{\circ} \mathrm{C}$ for 30 minutes. After that, the perovskite layer is prepared by spincoating the perovskite solution on the top of PEDOT:PSS in a nitrogen-filled glove box and transferred to a controlled humidity chamber where it is annealed at $85^{\circ} \mathrm{C}$ for 2 hours. $\mathrm{TiO}_{2}$ (an electron transporting layer material) is then spincoated on the perovskite film layer at $2500 \mathrm{rpm}$ for $30 \mathrm{~s}$ in a $\mathrm{N}_{2}$-filled glovebox. After spin-coating, the film was allowed to dry for about 18 hours. Ultimately, approximately $110 \mathrm{~nm}$ thick silver electrode was thermally deposited on the top of $\mathrm{TiO}_{2}$ layer in a vacuum with a base pressure of 0.000008 mbar and this step completes the fabrication of the PSCD. Throughout the entire device fabrication, we kept other process parameters constant while we varied humidity conditions in the chamber necessary for annealing the perovskite absorber layer.

2.5. Characterizations. The current density-voltage $(J-V)$ characteristics of PSCDs were measured using a Keithley 2400 source measurement unit scanning the devices at $100 \mathrm{mV} / \mathrm{s}$ and a Newport solar simulator with Air Mass 1.5 Global (AM1.5G) illumination and light intensity $100 \mathrm{~mW} / \mathrm{cm}^{2}$. Investigating the surface morphology and cross sections of our selected devices were carried out using a field emission scanning electron microscope (FESEM, JEOL7401). The incident photon-to-current conversion efficiency (IPCE) spectra was recorded over the wavelength from 300 to $800 \mathrm{~nm}$. The devices fabricated were tested under ambient environmental conditions with controlled humidity level and also under vacuum condition. The active device area was about $0.1225 \mathrm{~cm}^{2}$ after masking for $J-V$ measurements.

2.6. Device Architecture. Generally, there are two device architectures that are often used in the fabrication of halide perovskite structures, namely, planar heterojunction architecture (PHJ) and mesoscopic architecture [12, 13]. In our device architecture, we considered the PHJ architecture for the PSCD structure glass/ITO/PEDOT:PSS/ $\mathrm{MAPbI}_{3} / \mathrm{TiO}_{2} / \mathrm{Ag}$ (structure is stacked from bottom to top); the structure is seen in Figure 1. The PHJ-PSCD structure provides an interface that makes it easy to investigate material properties in the absence of the mesoporous scaffold layer. The energy level (in $\mathrm{eV}$ ) diagram of the corresponding 
TABLE 1: Device performance parameters summary.

\begin{tabular}{lcccc}
\hline Conditions & Voc $(\mathrm{V})$ & Jsc $\left(\mathrm{mA} / \mathrm{cm}^{2}\right)$ & FF $(\%)$ & PCE $(\%)$ \\
\hline Vacuum & 0.75 & 13.3 & 47.1 & 4.7 \\
$30 \% \mathrm{RH}$ & 0.77 & 13.9 & 53.1 & 5.6 \\
$60 \% \mathrm{RH}$ & 0.81 & 15.2 & 61.2 & 7.5 \\
$90 \% \mathrm{RH}$ & 0.73 & 13.8 & 46.6 & 4.5 \\
\hline
\end{tabular}
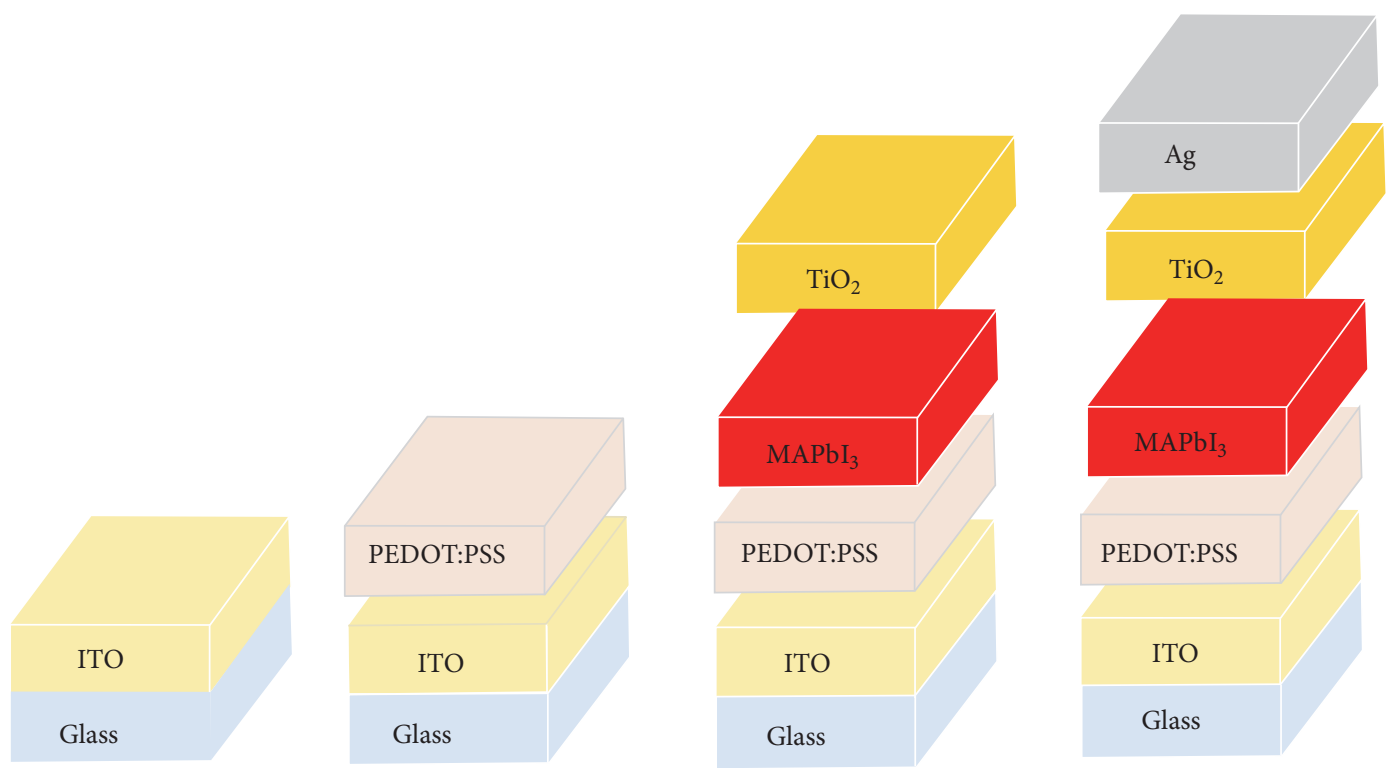

FIGURE 1: Device structure of the perovskite solar cell device.

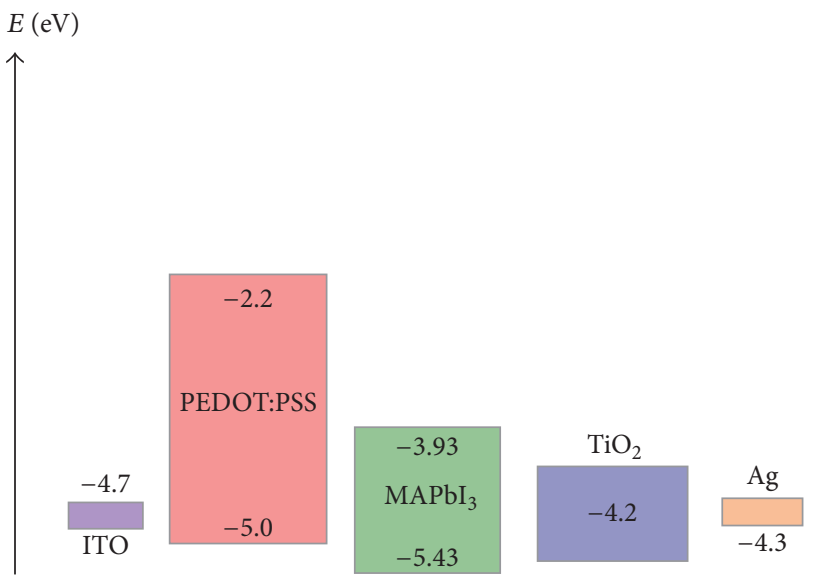

FIGURE 2: Corresponding energy level diagram in $\mathrm{eV}$ of the materials used in the perovskite solar cells.

materials used in the perovskite solar cells is depicted in Figure 2. The light source is shone from the glass/ITO end and it is important that the PEDOT:PSS layer is able to transmit the incoming light efficiently because this directly affects the generated current density. The transmittance of PEDOT:PSS is determined by the concentration of the spin-coated precursor solution; hence, it is important that we do not use a too high or too low PEDOT:PSS precursor solution.

\section{Results and Discussion}

The $\mathrm{PHJ}$ perovskite device structure is based on the p-i$n$ structure of ITO/PEDOT:PSS/MAPbI $/ 3 / \mathrm{TiO}_{2} / \mathrm{Ag}$. The ITO layer acts as the anode, PEDOT:PSS as the transparent and conductive layer for hole transport, $\mathrm{MAPbI}_{3}$ as the absorber material, $\mathrm{TiO}_{2}$ as the electron transport layer, and $\mathrm{Ag}$ as the cathode layer. The $J-V$ characteristics of the fabricated perovskite devices are shown in Figure 3(a). Table 1 shows the device performance of the four perovskite devices fabricated in four different humidity conditions. The perovskite device with the absorber material annealed at $60 \%$ relative humidity $(\mathrm{RH})$ showed high photovoltaic (PV) parameters with the maximum power conversion efficiency (PCE) of 10 device samples reaching 7.5\%. The device annealed under vacuum, $30 \%$ humidity, and $90 \%$ humidity conditions demonstrated efficiencies of $4.7 \%, 5.6 \%$, and $4.5 \%$, respectively. Further experiments were conducted to specifically test 10 perovskite devices annealed under $60-70 \%$ relative humidity before having to conclude that the optimal relative humidity was at $60 \%$. The maximum Voc (open-circuit voltage) and Jsc (short-circuit current density) are $0.81 \mathrm{~V}$ and $15.2 \mathrm{~mA} / \mathrm{cm}^{2}$. The largest fill factor derived was $61.2 \%$. According to Gangishetty et al. [14] low relative humidity will cause large gaps between perovskite crystallites and consequently leads to hysteresis that affects the overall performance of the device.

From Table 1, we observe a huge variation in the fill factor (FF) which can be attributed to the series resistance 
TABLE 2: Device performance parameters summary for 20 devices.

\begin{tabular}{|c|c|c|c|c|}
\hline Conditions & $\operatorname{Voc}(\mathrm{V})$ & $\mathrm{Jsc}\left(\mathrm{mA} / \mathrm{cm}^{2}\right)$ & $\mathrm{FF}(\%)$ & PCE (\%) \\
\hline \multirow{5}{*}{ Vacuum } & (1) 0.75 & 13.30 & 47.1 & 4.70 \\
\hline & (2) 0.77 & 13.10 & 46.5 & 4.69 \\
\hline & (3) 0.68 & 12.95 & 48.1 & 4.24 \\
\hline & (4) 0.70 & 13.02 & 47.5 & 4.33 \\
\hline & (5) 0.75 & 13.25 & 47.0 & 4.67 \\
\hline \multirow{5}{*}{$30 \% \mathrm{RH}$} & (6) 0.75 & 14.00 & 53.2 & 5.58 \\
\hline & (7) 0.75 & 13.95 & 53.2 & 5.57 \\
\hline & (8) 0.78 & 14.20 & 50.1 & 5.54 \\
\hline & (9) 0.78 & 14.30 & 50.1 & 5.58 \\
\hline & (10) 0.77 & 13.90 & 53.1 & 5.60 \\
\hline \multirow{5}{*}{$60 \% \mathrm{RH}$} & (11) 0.81 & 15.20 & 61.2 & 7.50 \\
\hline & (12) 0.78 & 14.55 & 61.3 & 6.96 \\
\hline & (13) 0.80 & 15.00 & 62.2 & 7.46 \\
\hline & (14) 0.80 & 15.00 & 61.5 & 7.38 \\
\hline & (15) 0.80 & 14.67 & 61.0 & 7.16 \\
\hline \multirow{5}{*}{$90 \% \mathrm{RH}$} & (16) 0.73 & 13.80 & 46.6 & 4.59 \\
\hline & (17) 0.70 & 13.70 & 46.8 & 4.48 \\
\hline & (18) 0.71 & 13.67 & 46.4 & 4.50 \\
\hline & (19) 0.65 & 13.40 & 49.5 & 4.31 \\
\hline & (20) 0.68 & 13.80 & 47.5 & 4.46 \\
\hline
\end{tabular}

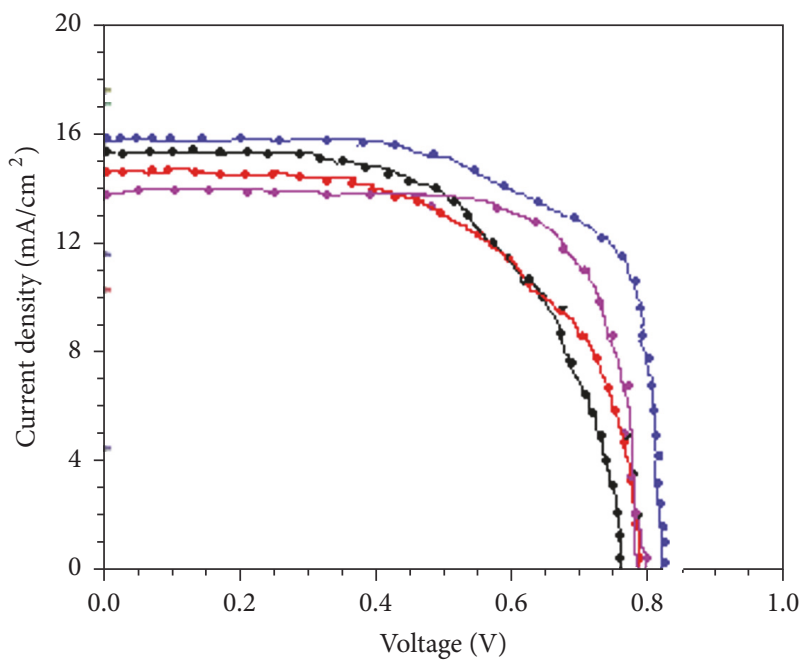

(a) Typical $J$ - $V$ characteristics of four perovskite devices fabricated under different atmospheric conditions. Red: vacuum, black: $30 \% \mathrm{RH}$, blue: $60 \%$ RH condition, and violet: $90 \% \mathrm{RH}$

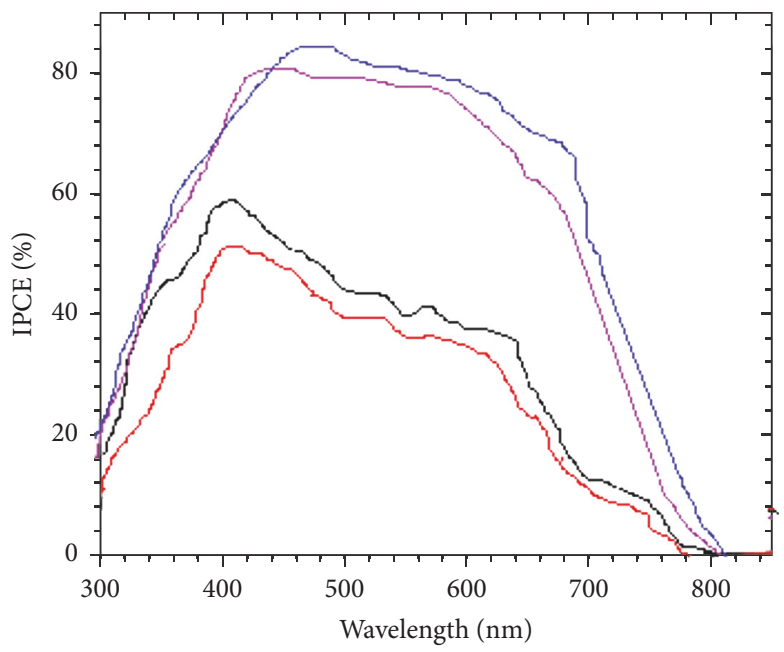

(b) IPCE spectra of four perovskite devices fabricated under different atmospheric conditions. Red: vacuum, black: $30 \% \mathrm{RH}$, blue: $60 \% \mathrm{RH}$ condition, and violet: $90 \% \mathrm{RH}$

Figure 3

of the devices which is primarily due to the charge transport properties of the charge carrier properties of HTM [15]. The smaller resistance present in the $60 \% \mathrm{RH}$ condition likely contributed to the higher FF value. On top of that, Voc values experience a big gap when compared with other conditions; this is very possible when fewer bimolecular recombinations occur as a result of reduced number of traps and grain boundaries [12]. The IPCE spectra supports the evident increase in the short-circuit current density as seen in Figure 3(b). The measurements taken for IPCE were conducted over a spectral range of $300 \mathrm{~nm}$ to $800 \mathrm{~nm}$.

The statistical distributions of the PCE of 20 fabricated perovskite device samples are shown in Figure 4. Table 2 also shows the different values of Voc, Jsc, FF, and PCE for 20 perovskite solar cell devices fabricated in vacuum, $30 \% \mathrm{RH}$, $60 \% \mathrm{RH}$, and $90 \% \mathrm{RH}$. Table 3 is the best cell performance 
TABLE 3: Best device performance for different scan direction and scan rate.

\begin{tabular}{|c|c|c|c|c|c|c|}
\hline Conditions & Scan direction & Scan rate $(\mathrm{V} / \mathrm{s})$ & $\operatorname{Voc}(\mathrm{V})$ & $\mathrm{Jsc}\left(\mathrm{mA} / \mathrm{cm}^{2}\right)$ & FF (\%) & PCE (\%) \\
\hline \multirow{8}{*}{ Vacuum } & \multirow{4}{*}{ Reverse scan } & 0.05 & 0.72 & 12.32 & 44.2 & 3.92 \\
\hline & & 0.1 & 0.74 & 12.57 & 45.0 & 4.19 \\
\hline & & 0.2 & 0.74 & 12.94 & 45.6 & 4.37 \\
\hline & & 0.5 & 0.75 & 13.30 & 47.1 & 4.70 \\
\hline & \multirow{4}{*}{ Forward scan } & 0.05 & 0.72 & 12.30 & 44.2 & 3.91 \\
\hline & & 0.1 & 0.74 & 12.55 & 44.9 & 4.17 \\
\hline & & 0.2 & 0.74 & 12.94 & 45.6 & 4.37 \\
\hline & & 0.5 & 0.75 & 13.29 & 46.9 & 4.67 \\
\hline \multirow{8}{*}{$30 \% \mathrm{RH}$} & \multirow{4}{*}{ Reverse scan } & 0.05 & 0.73 & 12.65 & 51.6 & 4.76 \\
\hline & & 0.1 & 0.75 & 12.95 & 52.2 & 5.07 \\
\hline & & 0.2 & 0.76 & 13.45 & 52.8 & 5.39 \\
\hline & & 0.5 & 0.77 & 13.90 & 53.1 & 5.68 \\
\hline & \multirow{4}{*}{ Forward scan } & 0.05 & 0.73 & 12.62 & 51.1 & 4.71 \\
\hline & & 0.1 & 0.74 & 13.40 & 52.0 & 5.16 \\
\hline & & 0.2 & 0.75 & 13.45 & 52.5 & 5.30 \\
\hline & & 0.5 & 0.76 & 13.90 & 52.8 & 5.57 \\
\hline \multirow{8}{*}{$60 \% \mathrm{RH}$} & \multirow{4}{*}{ Reverse scan } & 0.05 & 0.77 & 14.00 & 59.5 & 6.41 \\
\hline & & 0.1 & 0.79 & 14.28 & 60.1 & 6.78 \\
\hline & & 0.2 & 0.79 & 14.78 & 60.7 & 7.08 \\
\hline & & 0.5 & 0.81 & 15.20 & 61.2 & 7.50 \\
\hline & \multirow{4}{*}{ Forward scan } & 0.05 & 0.77 & 13.90 & 59.1 & 6.33 \\
\hline & & 0.1 & 0.77 & 14.25 & 60.0 & 6.58 \\
\hline & & 0.2 & 0.78 & 14.70 & 60.4 & 6.93 \\
\hline & & 0.5 & 0.81 & 15.19 & 60.8 & 7.48 \\
\hline \multirow{8}{*}{$90 \% \mathrm{RH}$} & \multirow{4}{*}{ Reverse scan } & 0.05 & 0.70 & 13.10 & 45.2 & 4.14 \\
\hline & & 0.1 & 0.71 & 13.20 & 45.6 & 4.27 \\
\hline & & 0.2 & 0.72 & 13.45 & 46.0 & 4.45 \\
\hline & & 0.5 & 0.73 & 13.80 & 46.6 & 4.59 \\
\hline & \multirow{4}{*}{ Forward scan } & 0.05 & 0.69 & 12.80 & 44.5 & 3.93 \\
\hline & & 0.1 & 0.70 & 12.80 & 44.9 & 4.02 \\
\hline & & 0.2 & 0.71 & 13.35 & 45.4 & 4.30 \\
\hline & & 0.5 & 0.73 & 13.80 & 46.0 & 4.53 \\
\hline
\end{tabular}

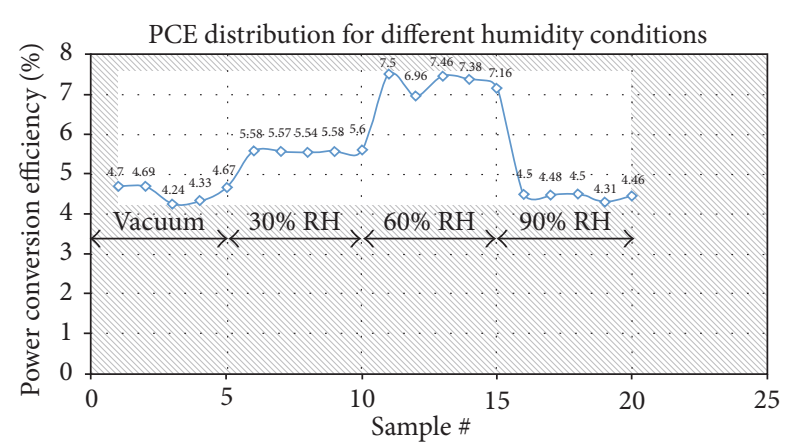

FIGURE 4: PCE distribution of twenty perovskite devices fabricated under different atmospheric conditions (vacuum, 30\% RH, 60\% RH, and $90 \% \mathrm{RH})$.

characteristics table for different humidity conditions with respect to scan direction and rate.
Figure 5 shows the PV characteristics properties of the $\mathrm{MAPbI}_{3}$ hybrid perovskite solar cell. The $J-V$ curve of the best performing $\mathrm{MAPbI}_{3}$ hybrid perovskite solar cell is the one fabricated under $60 \% \mathrm{RH}$ condition, Voc of $0.81 \mathrm{~V}$, Jsc of $15.20 \mathrm{~mA} / \mathrm{cm}^{2}, \mathrm{FF}$ of $61.2 \%$, and PCE of $7.5 \%$ in the reverse scan direction, and Voc of $0.81 \mathrm{~V}$, Jsc of $15.19 \mathrm{~mA} / \mathrm{cm}^{2}, \mathrm{FF}$ of $60.8 \%$, and PCE of $7.48 \%$ in the forward scan direction under standard illumination of $100 \mathrm{~mW} / \mathrm{cm}^{2}$ (AM1.5 global). According to the $J-V$ curves, the $\mathrm{MAPbI}_{3}$ hybrid perovskite cell showed a very small $J-V$ hysteresis with respect to the scan direction and a significant $J-V$ hysteresis is observed when the scan rate is varied from $0.05 \mathrm{~V} / \mathrm{s}$ to $0.5 \mathrm{~V} / \mathrm{s}$.

The X-ray diffraction (XRD) patterns of the perovskite films under different annealing conditions (vacuum, 30\% RH, $60 \% \mathrm{RH}$, and $90 \% \mathrm{RH}$ ) are shown in Figure 6. XRD spectra results for each film as deposited on ITO/PEDOT:PSS are reported in order to check the crystallinity of perovskites. The main diffraction peaks of the perovskite films are observed at $14.1^{\circ}$ and $28.4^{\circ}$. A new diffraction peak is observed at 


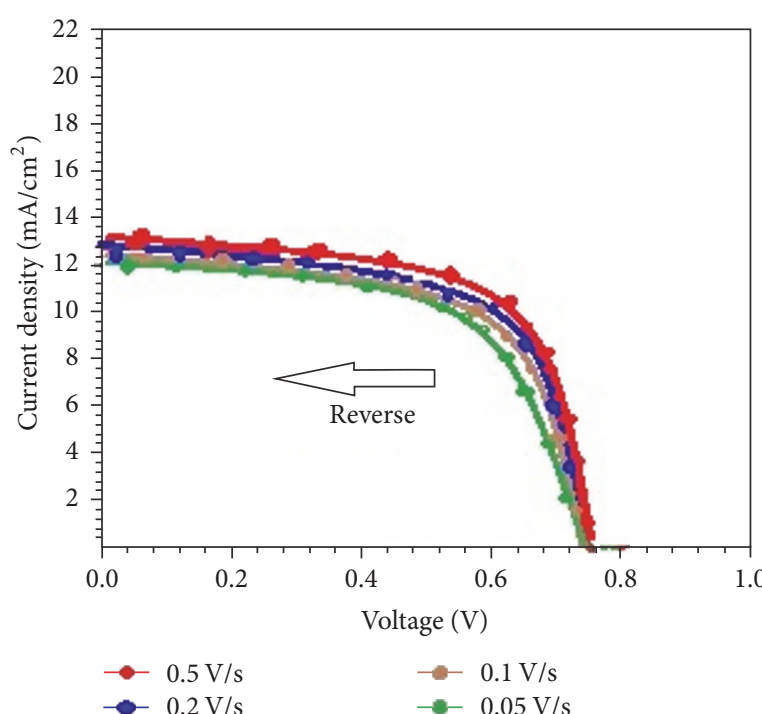

(a) Vacuum (reverse scan)

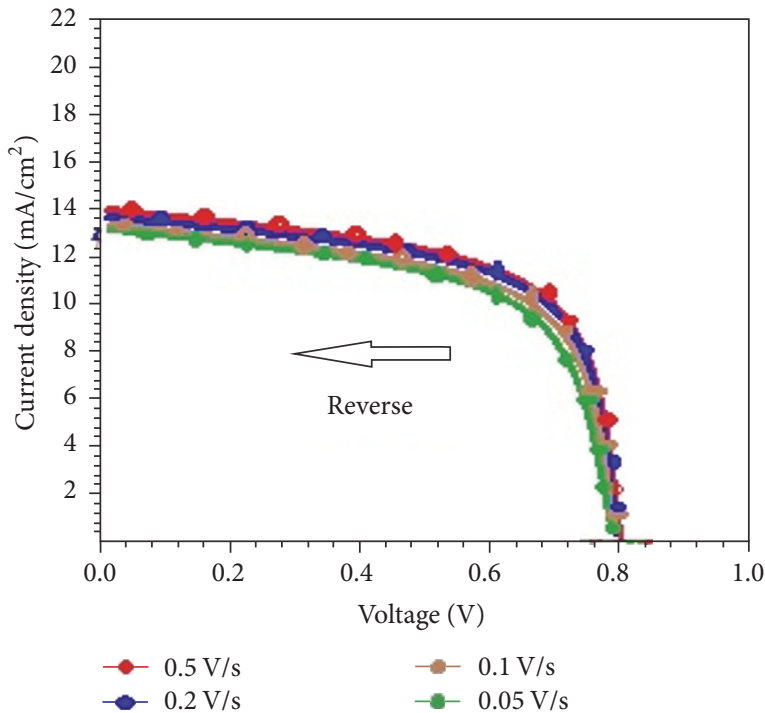

(c) $30 \% \mathrm{RH}$ (reverse scan)

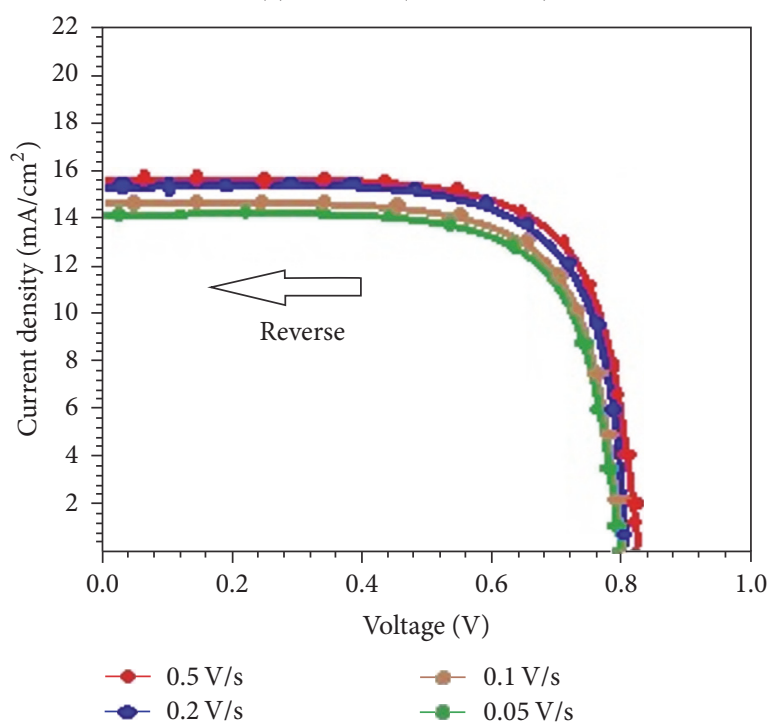

(e) $60 \% \mathrm{RH}$ (reverse scan)

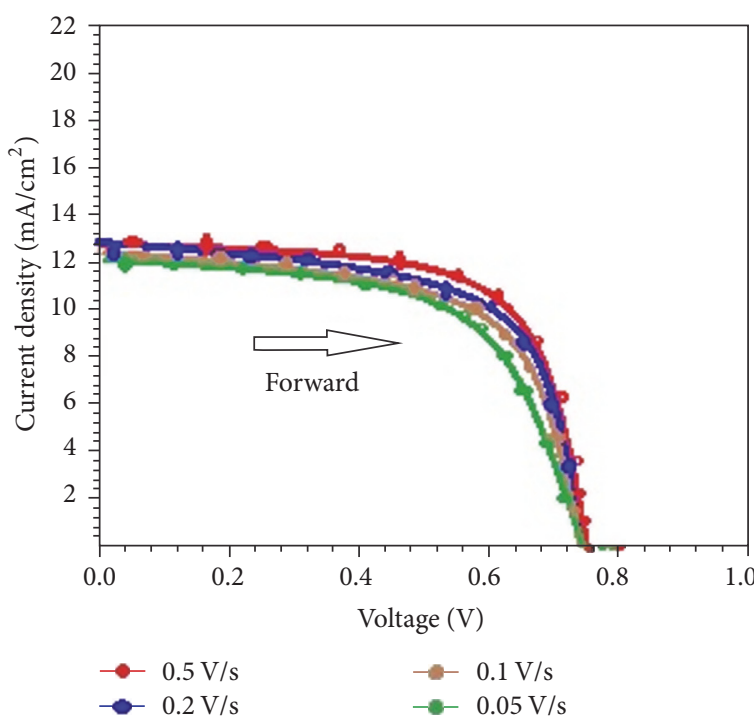

(b) Vacuum (forward scan)

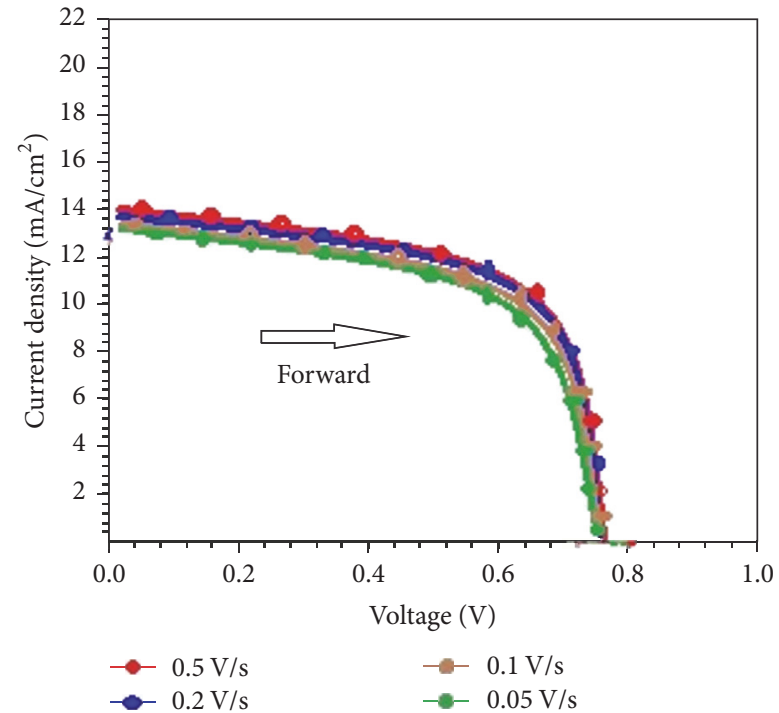

(d) $30 \% \mathrm{RH}$ (forward scan)

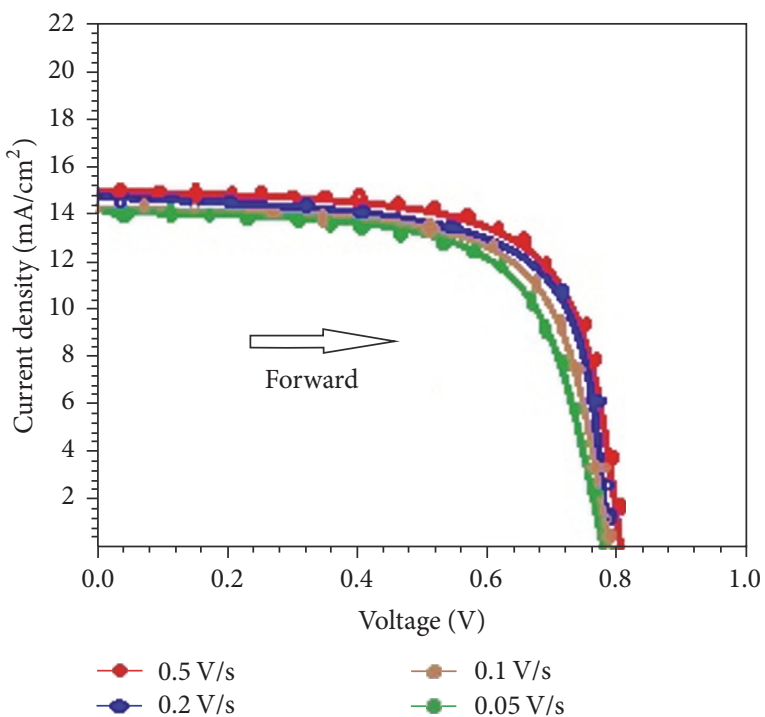

(f) $60 \% \mathrm{RH}$ (forward scan)

Figure 5: Continued. 


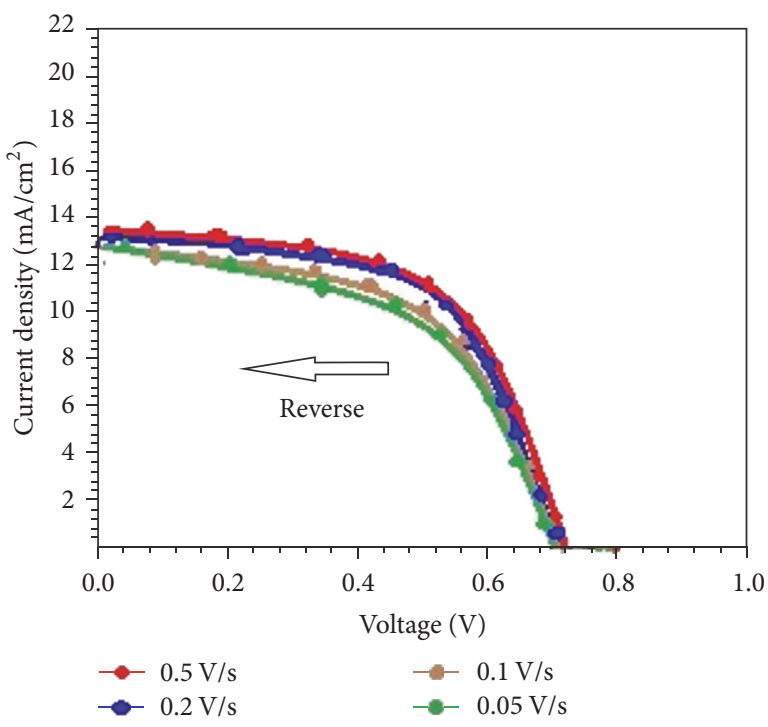

(g) $90 \% \mathrm{RH}$ (reverse scan)

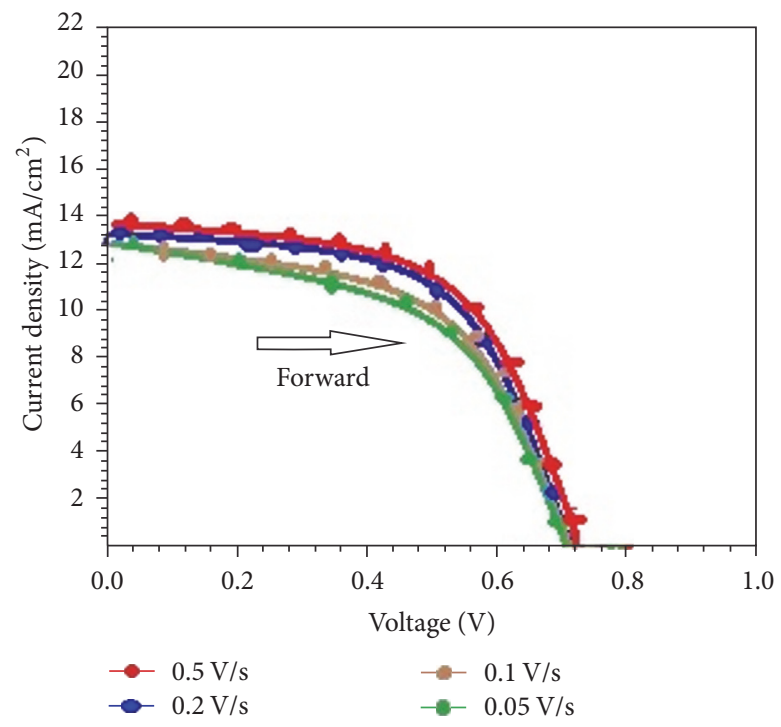

(h) $90 \% \mathrm{RH}$ (forward scan)

FiguRE 5: $J$ - $V$ curves of the $\mathrm{MAPbI}_{3}$ hybrid perovskite solar cell with respect to different scan directions (reverse and forward) and different scan rates $(0.05 \mathrm{~V} / \mathrm{s}, 0.1 \mathrm{~V} / \mathrm{s}, 0.2 \mathrm{~V} / \mathrm{s}$, and $0.5 \mathrm{~V} / \mathrm{s})$.

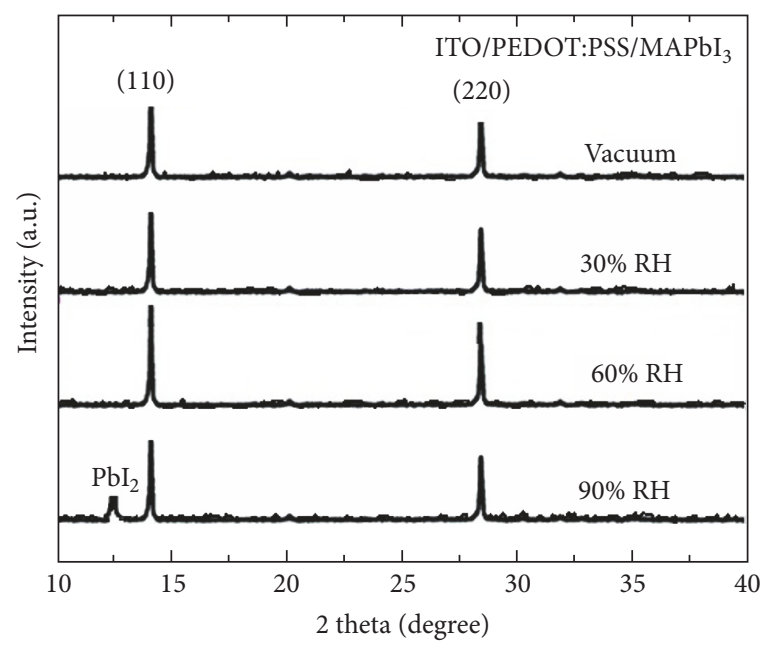

FIGURE 6: XRD patterns of perovskite films annealed in different conditions (vacuum, 30\% RH, 60\% RH, and 90\% RH).

$12.6^{\circ}$ in the XRD spectra of $90 \% \mathrm{RH}$; this can be attributed to the diffraction peak of $\mathrm{PBI}_{2}$ formed during the decomposition of perovskite crystal structure when exposed to excess moisture and oxygen. The main difference observed in the other annealing conditions is simply the minimal increase in the peak intensity (from vacuum to $60 \% \mathrm{RH}$ ). This demonstrates that there is a boost in the crystallinity of $\mathrm{MAPbI}_{3}$ films formed in the presence of increased relative humidity conditions.

The perovskite film was spin-coated using two-deposition technique [16]. Obtaining an excellent performance in $\mathrm{PHJ}$ perovskite devices requires that we obtain a quality film morphology by way of how the spin-coated film is annealed and crystallized. The rate of crystallization of perovskite absorber film is one of the many difficult parameters to be directly controlled in the fabrication process; we decide to understand the crystallization behavior of the film by taking SEM images whereby we are able to understand the surface morphological features peculiar to the film under study. The crystallinity of the film influences factors such as diffusion length, hole and electron mobility, and charge dissociation efficiency [3,17]. The crystallization behavior of perovskite material as seen in the results from SEM images shows that humidity is a major factor contributing to the way perovskite crystals are formed. Zhou et al. [18] showed that mixed halide perovskite when annealed under $30 \% \mathrm{RH}$ was subject to a different conversion process; $\mathrm{MAPbI}_{3}$ phase could be observed distinctly in the presence of moisture. 


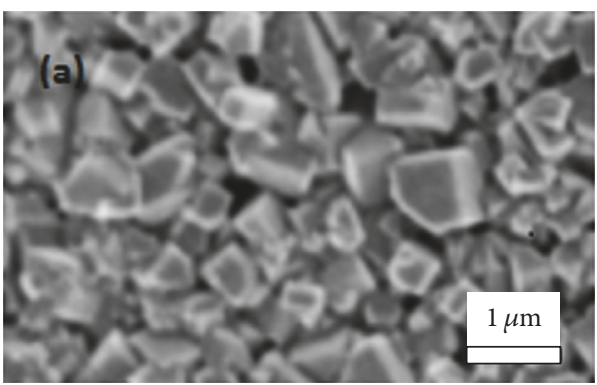

(a)

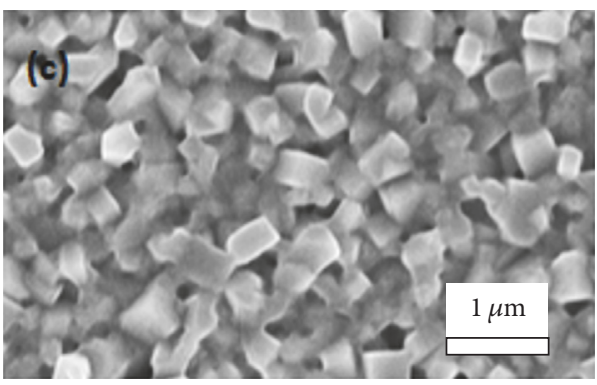

(c)

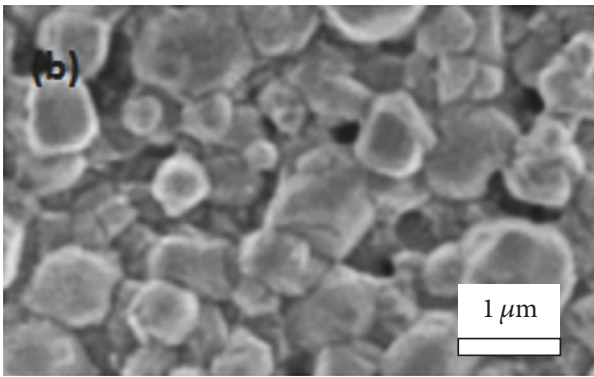

(b)

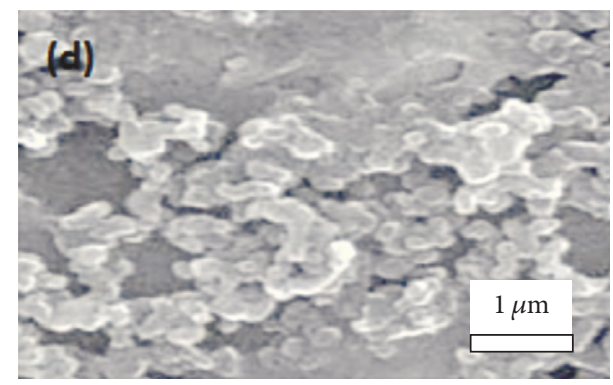

(d)

Figure 7: SEM images of perovskite absorber in (a) vacuum, (b) $30 \% \mathrm{RH}$, (c) $60 \% \mathrm{RH}$, and (d) $90 \% \mathrm{RH}$.

Figure 7 shows the SEM images of perovskite $\left(\mathrm{MAPbI}_{3}\right)$ formed by two-step deposition of $\mathrm{PbI}_{2}$ and the precursor solution. Here we show the annealing treatment for the perovskite absorber carried out at different relative humidity $(0 \%, 30 \%, 60 \%$, and $90 \%)$. We observed the formation of cuboid crystals across all the humidity conditions but varying number of pin-holes (trap states). The greatest number of pinholes is seen in the $90 \% \mathrm{RH}$, next to that is vacuum, next to that is $30 \% \mathrm{RH}$, and finally with the least number of pin-holes is $60 \% \mathrm{RH}$. There is a linear relationship existing between the number of pin-holes in the film and also the power conversion efficiency of the perovskite device; this explains the reason for high efficiency in the $60 \% \mathrm{RH}$ condition (because the cuboid crystals are tightly packed with very few gaps present).

\section{Conclusion}

In this work, we developed an effective condition for annealing perovskite absorber after spin-coating using humidity control experimental methods to determine the effect of environmental conditions (humidity and moisture) on the perovskite film and the performance of the organic-inorganic halide perovskite $\mathrm{MAPbI}_{3}$ solar device. PEDOT:PSS is used as the HTM and $\mathrm{TiO}_{2}$ as the ETM. It is important that we carefully select the transporting layers as this plays a vital role in optimizing the overall power conversion efficiency and performance. It was found out that the efficiencies of the PSCDs are highly dependent on the crystallization and annealing environment of the perovskite absorber, as the relative humidity falls in the range $\sim 60-65 \%$; we observe a significant improvement in the morphology of $\mathrm{MAPbI}_{3}$ due to formation of more uniform perovskite crystallites with fewer grain boundaries.

Additionally, efficiency improvements can be achieved through proper selection of absorber, HTL, and ETL materials and cathode/anode electrode material, controlling film thickness, and interface engineering. Efficiency of up to $7.5 \%$ was achieved using a normal PHJ structure solar cell. The results presented show a promising progress towards achieving high-efficiency photovoltaic device applications.

\section{Conflicts of Interest}

The authors declare that they have no conflicts of interest.

\section{References}

[1] Y.-C. Huang, C.-S. Tsao, Y.-J. Cho et al., "Insight into evolution, processing and performance of multi-length-scale structures in planar heterojunction perovskite solar cells," Scientific Reports, vol. 5, Article ID 13657, 2015.

[2] T. Salim, S. Sun, Y. Abe et al., "Perovskite-based solar cells: impact of morphology and device architecture on device performance," Journal of Materials Chemistry A, no. 17, pp. 8943-8969, 2015.

[3] G. E. Eperon, V. M. Burlakov, P. Docampo, A. Goriely, and H. J. Snaith, "Morphological control for high performance, solution-processed planar heterojunction perovskite solar cells," Advanced Functional Materials, vol. 24, no. 1, pp. 151157, 2014.

[4] X. Gong, M. Li, X.-B. Shi, H. Ma, Z.-K. Wang, and L.-S. Liao, "Controllable perovskite crystallization by water additive for high-performance solar cells," Advanced Functional Materials, vol. 25, no. 42, pp. 6671-6678, 2015. 
[5] H.-L. Hsu, C.-P. Chen, J.-Y. Chang, Y.-Y. Yu, and Y.-K. Shen, "Two-step thermal annealing improves the morphology of spin-coated films for highly efficient perovskite hybrid photovoltaics," Nanoscale, vol. 6, no. 17, pp. 10281-10288, 2014.

[6] A. Dualeh, N. Tétreault, T. Moehl, P. Gao, M. K. Nazeeruddin, and M. Grätzel, "Effect of annealing temperature on film morphology of organic-inorganic hybrid pervoskite solid-state solar cells," Advanced Functional Materials, vol. 24, no. 21, pp. 3250-3258, 2014.

[7] M. Saliba, K. W. Tan, H. Sai et al., "Influence of thermal processing protocol upon the crystallization and photovoltaic performance of organic-inorganic lead trihalide perovskites," Journal of Physical Chemistry C, vol. 118, no. 30, pp. 17171-17177, 2014.

[8] Y. Tidhar, E. Edri, H. Weissman et al., "Crystallization of methyl ammonium lead halide perovskites: implications for photovoltaic applications," Journal of the American Chemical Society, vol. 136, no. 38, pp. 13249-13256, 2014.

[9] G. Grancini, S. Marras, M. Prato et al., "The impact of the crystallization processes on the structural and optical properties of hybrid perovskite films for photovoltaics," Journal of Physical Chemistry Letters, vol. 5, no. 21, pp. 3836-3842, 2014.

[10] P.-W. Liang, C.-Y. Liao, C.-C. Chueh et al., "Additive enhanced crystallization of solution-processed perovskite for highly efficient planar-heterojunction solar cells," Advanced Materials, vol. 26, no. 22, pp. 3748-3754, 2014.

[11] J.-H. Im, C.-R. Lee, J.-W. Lee, S.-W. Park, and N.-G. Park, "6.5\% Efficient perovskite quantum-dot-sensitized solar cell," Nanoscale, vol. 3, no. 10, pp. 4088-4093, 2011.

[12] Y. Shao, Z. Xiao, C. Bi, Y. Yuan, and J. Huang, "Origin and elimination of photocurrent hysteresis by fullerene passivation in $\mathrm{CH}_{3} \mathrm{NH}_{3} \mathrm{PbI}_{3}$ planar heterojunction solar cells," Nature Communications, vol. 5, article 5784, 2014.

[13] M. M. Lee, J. Teuscher, T. Miyasaka, T. N. Murakami, and H. J. Snaith, "Efficient hybrid solar cells based on mesosuperstructured organometal halide perovskites," Science, vol. 338, no. 6107, pp. 643-647, 2012.

[14] M. K. Gangishetty, R. W. J. Scott, and T. L. Kelly, "Effect of relative humidity on crystal growth, device performance and hysteresis in planar heterojunction perovskite solar cells," Nanoscale, vol. 8, no. 12, pp. 6300-6307, 2016.

[15] A. D. Sheikh et al., "Atmospheric effects on the photovoltaic performance of hybrid perovskite solar cells," Solar Energy Materials \& Solar Cells, vol. 137, pp. 6-14, 2015.

[16] J. Burschka, N. Pellet, S. Moon et al., "Sequential deposition as a route to high-performance perovskite-sensitized solar cells," Nature, vol. 499, no. 7458, pp. 316-319, 2013.

[17] G. Xing, N. Mathews, S. Sun et al., "Long-range balanced electron- and hole-transport lengths in organic-inorganic $\mathrm{CH}_{3} \mathrm{NH}_{3} \mathrm{PbI}_{3}$," Science, vol. 342, pp. 344-347, 2013.

[18] H. Zhou, Q. Chen, G. Li et al., "Interface engineering of highly efficient perovskite solar cells," Science, vol. 345, no. 6196, pp. 542-546, 2014. 


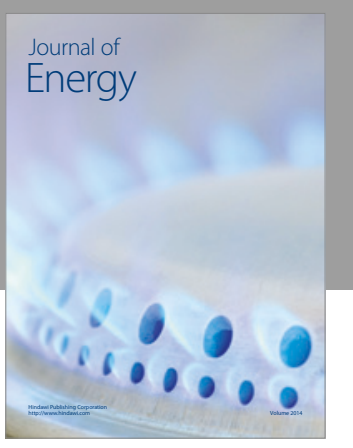

Journal of

Industrial Engineering
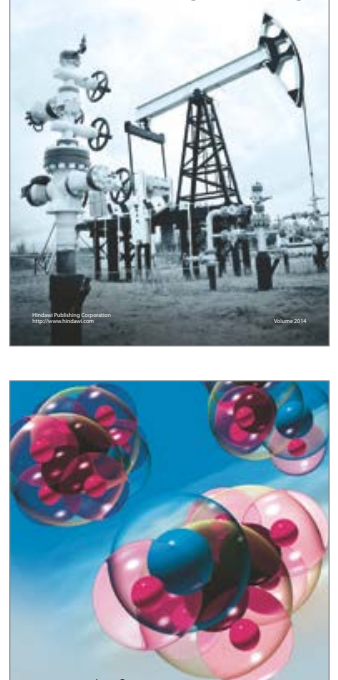

Fuels
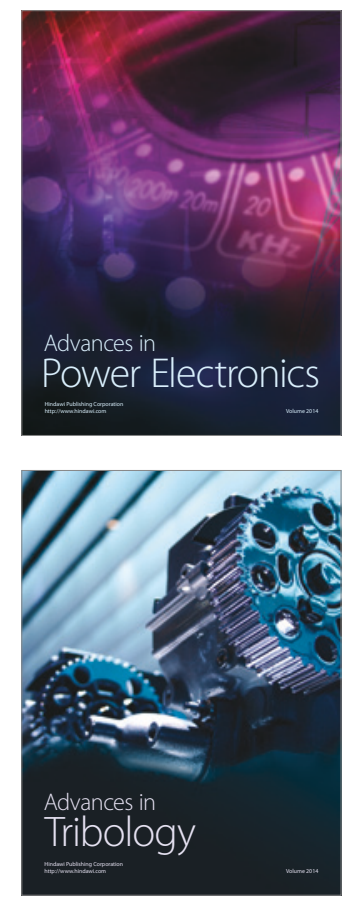
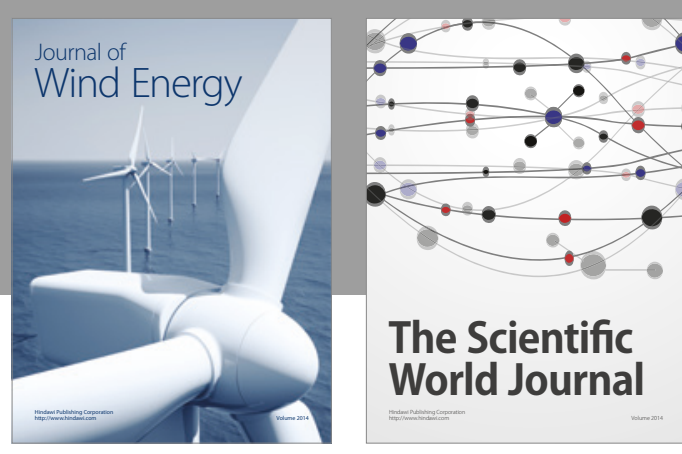

The Scientific World Journal
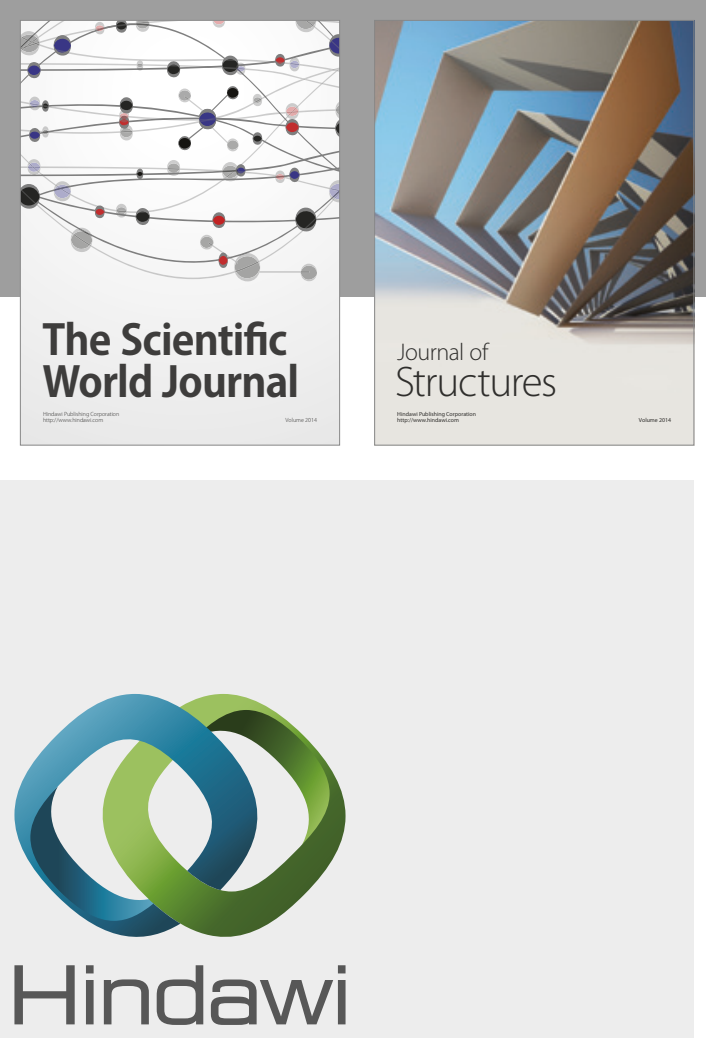

Submit your manuscripts at

https://www.hindawi.com
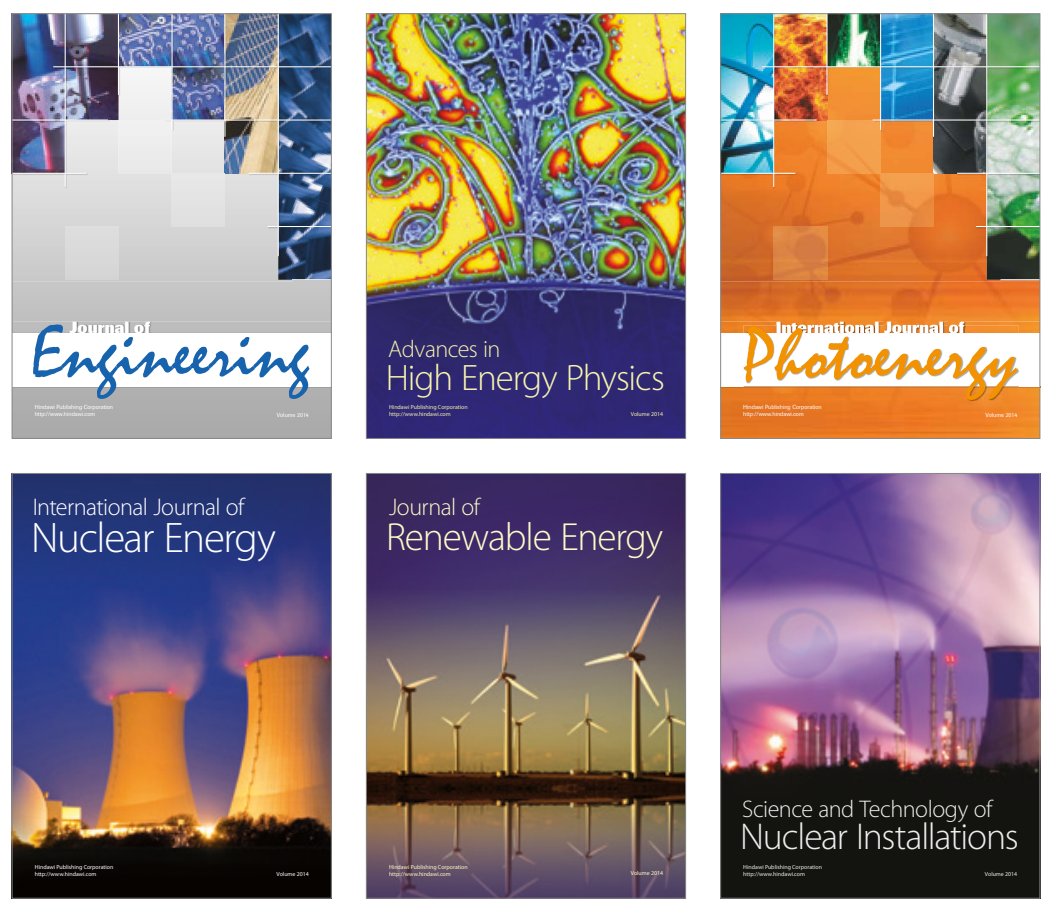

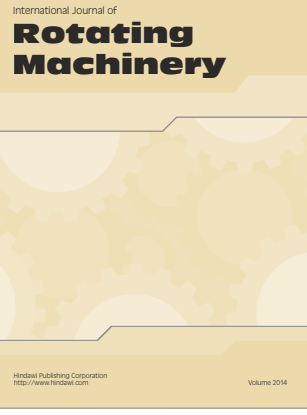

Journal of

Petroleum Engineering

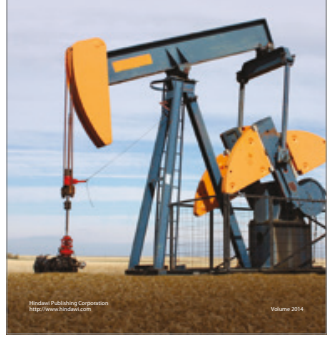

Journal of
Solar Energy
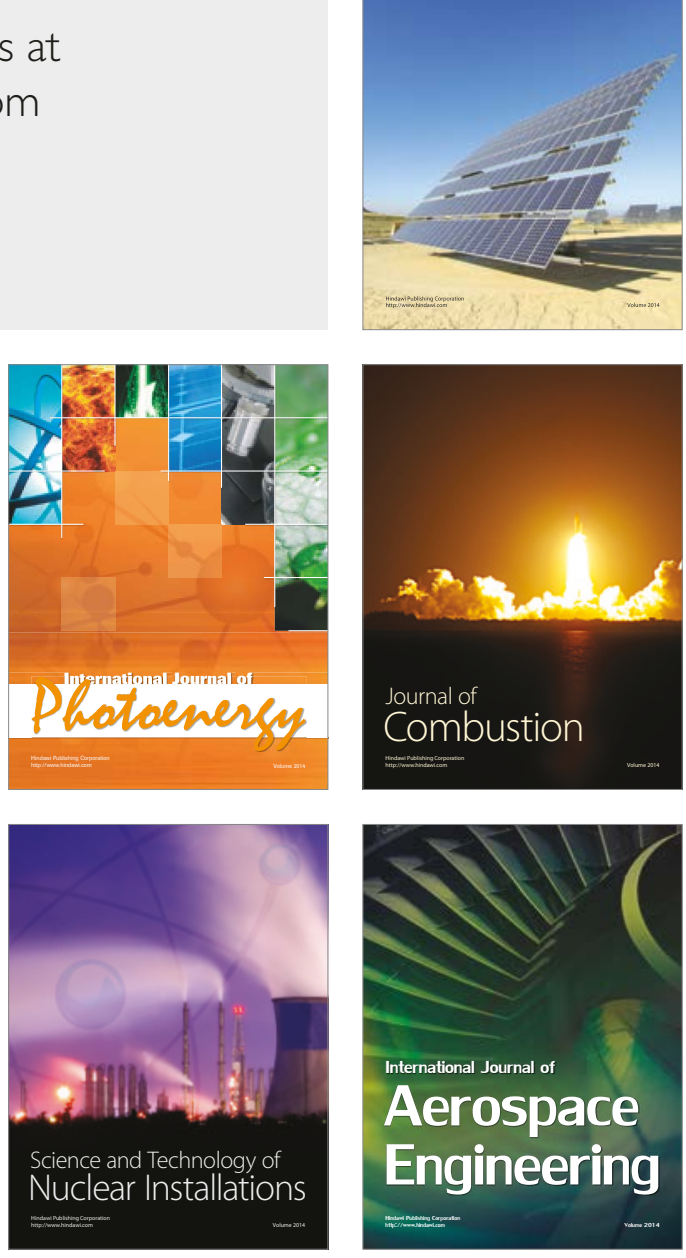